\title{
On the occasion of publication of the feature on differential diagnosis and treatment of hepatocellular carcinoma: the role of ultrasound
}

\author{
Kazushi Numata ${ }^{1}$
}

() The Japan Society of Ultrasonics in Medicine 2020

Liver cancer is the 6th most common cancer in terms of incidence and has the 4th highest cancer-related mortality around the world [1]. Ninety percent of liver cancer is hepatocellular carcinoma (HCC). Compared with other regions, Japan has achieved highly favorable outcomes in HCC treatment [2]. Notable regional differences were observed in the time between initial diagnosis and death. The median survival of patients with Barcelona Clinic Liver Cancer (BCLC) stage A HCCs (curatively treatable early-stage disease) was 91.0 months in Japan, which is considerably longer than that in other regions (54.0 months in the Asia-Pacific region, 49.3 months in Europe, 23.3 months in Latin America, and 24.9 months in the United States), although each BCLC stage group is expected to consist of homogeneous patients in terms of tumor burden and liver function [3, 4].

Several factors have contributed to this achievement. For example, Japan was the first country in the world to develop and implement diagnostic ultrasound (US) systems for liver cancer screening [2]. The health insurance system in Japan has been maintained well, allowing anyone to visit a medical institution. Furthermore, the penetration rate of diagnostic imaging equipment such as US, computed tomography (CT), and magnetic resonance imaging (MRI) is higher than in other countries, enabling more accurate and early diagnosis of small HCCs. These factors seem to be linked to better outcomes of overall survival for patients with early-stage HCCs.

Additionally, Sonazoid (GE Healthcare, Amersham, UK), perfluorobutane microbubbles coated with hydrogenated phosphatidylserine as a contrast-enhanced US (CEUS) agent, was approved in Japan for the imaging of hepatic lesions in 2007. This contrast agent was also approved in South Korea in 2012 and in Taiwan in 2017, and it will be

Kazushi Numata

kz-numa@urahp.yokohama-cu.ac.jp

1 Gastroenterological Center, Yokohama City University Medical Center, Urafune-cho 4-57, Minami-ku, Yokohama, Kanagawa 232-0024, Japan approved in China for focal hepatic lesion. Unlike the strict intravascular imaging of SonoVue (Bracco, Milan, Italy), Sonazoid is taken up by Kupffer cells. About 10 min after Sonazoid injection, in the post-vascular (Kupffer) phase, the sonographer can continue to observe the hepatic lesion, especially for information that is not clearly obtained in the arterial or portal phase, because hepatic lesions having decreased or no Kupffer cells appear as hypoechoic during the post-vascular phase. Hypervascular HCCs with an isoechoic or unclear margin on conventional US, in particular, appear as hypoechoic during the post-vascular phase, and we can puncture them in real time. CEUS using Sonazoid is useful for guidance of percutaneous ablation therapy of HCCs not detected by conventional US [5].

For the detection of HCCs, a combination of modalities using different imaging techniques is recommended to increase the sensitivity and specificity of diagnosis. Since 2009, "fusion imaging," which fuses US images with multiplanar reconstructed CT or MRI images on a single screen in real time, has become available. Fusion imaging combining conventional US and the hepatobiliary phase (HBP) of contrast-enhanced MRI with gadolinium ethoxybenzyl diethylenetriaminepentaacetic acid (Gd-EOB-DTPA, Primovist; Bayer Schering Pharma AG, Berlin, Germany; EOB-MRI) as the reference standard can help to select targeted HCC lesions, especially early HCC lesions that appear as hypo- or hyperechoic lesions on conventional US [6]. After confirmation of the location of the target lesion, the vascularity of each hepatic lesion can then be evaluated using CEUS with Sonazoid. Moreover, we can perform biopsy from the target lesion detected by fusion imaging combining B-mode or CEUS and HBP EOBMRI.

Recently, a new Doppler microvascular imaging technique, Superb Microvascular Imaging (SMI; Canon Medical Systems, Otawara, Japan) was introduced clinically. This innovative vascular imaging technique can detect microvascular flow at low velocity without contrast enhancement. SMI removes tissue motion and extracts flow signals, and the 
vascular information is then displayed as a monochrome or color map of flow. SMI was superior to color Doppler flow imaging in detecting microvascular blood flow signals. More hypervascular supply patterns were depicted in HCCs than in non-HCCs, suggesting a promising diagnostic value for SMI in the differentiation between HCCs and non-HCCs [7].

Regarding treatment of HCCs, radiofrequency ablation (RFA) is widely used for achievement of complete necrosis of HCCs. A three-dimensional (3D) display method, a 3D global positioning system (GPS) marker that is one of the functions of volume navigation (V Nav; GE Healthcare, Chalfont St. Giles, UK), was recently introduced in clinical practice. This 3D-GPS marker may easily change the size and shape of an ellipse. Using this function, operators may make more accurate simulations of how they ablate the tumor with sufficient margins in three dimensions. However, it should be noted that the positional information derived by these 3D-GPS markers is not entirely consistent due to respiratory fluctuations, among other factors.

The ablation margin immediately after RFA can be hard to determine by CEUS alone because of the difficulty in identifying the edge between the ablated HCC and the ablated adjacent liver parenchyma using this imaging modality alone. Fusion imaging combining CEUS and arterialphase CECT as a reference appears to be a useful method for evaluating the therapeutic efficacy of RFA for hypervascular HCCs detected using conventional US [8]. Fusion imaging combining CEUS and HBP EOBMRI also appears to be useful for evaluating the therapeutic efficacy of RFA for HCCs identified as having isoechoic or unclear margins on conventional US [9].

However, a fusion image does not always precisely correspond to the US image. One of the reasons for registration error is the difference in timing of breath-holding during image acquisition by each modality. Therefore, the image registration error associated with use of CEUS/CECT or CEMRI fusion imaging can be reduced by US or CEUS/US fusion imaging, namely, real-time US or CEUS combined with pretreatment 3D US to obtain volume data, because the breath-hold time is almost the same $[10,11]$. The usefulness of US or CEUS/US fusion imaging for evaluation of the therapeutic efficacy of RFA has been reported previously $[10,11]$. However, the US volume data in that study were obtained by manual sweep 3D US. For a sonographer who is not familiar with that modality, it may be difficult to maintain a constant speed of scanning without the transducer slipping upon hitting a rib in manual-sweep 3D US, which may result in deformed volume data and, consequently, inaccurate evaluation of the therapeutic efficacy of RFA. In contrast, accurate US volume data can be obtained with 3D US in the auto sweep (AutoSweep; GE Healthcare, Ltd., Chicago, IL) because the sonographer can scan the tumor and surrounding liver parenchyma at a constant scanning speed [12]. CEUS/US (3D US volume data acquisition by AutoSweep) fusion imaging during RFA may be a useful method for guiding RFA as well as for evaluating its therapeutic efficacy [12].

Registration error is inevitable in any fusion imaging because of the changes in liver morphology caused by respiration and/or slight movements of the patient due to pain during RFA, and adjustments to fix the registration error between the two modalities are needed several times during RFA [12]. Registration error caused by respiratory movement is the biggest drawback of fusion imaging. For patients who cannot hold their breath sufficiently, it would be useful if a motion tracking system could be developed in the near future to compensate for the movements of the tumor with respiration so as to maintain concordance between CEUS and other US images [13].

A newly developed microwave ablation (MWA) system can ablate HCCs larger than $3 \mathrm{~cm}$ in maximum diameter in a short time even though adjacent to major vessels due to no heat-sink effect. Compared with RFA, this newly developed MWA system seems to be a promising treatment for large HCCs and liver metastases [14]. However, we have to take precautions against excessive ablation of adjacent tissues and organs. Evaluation of therapeutic efficacy using CECU may be useful to determine the ablative area immediately after MWA. With recent advances in chemotherapy, the concept of mass reduction born in colorectal cancer may also arise in HCCs in the near future. A combination of MWA, which can widely ablate central areas of HCCs in a short time, and RFA, which can safely ablate the marginal areas of HCCs, may be useful for mass reduction of large HCCs.

For this feature, Dr. Feiqian Wang explained (1) the differences between two commonly used CEUS agents, SonoVue and Sonazoid; (2) low mechanical index (MI) contrast imaging as a representative imaging method with Sonazoid, and high MI contrast imaging and low MI harmonic imaging as an alternative Sonazoid imaging method; (3) differential diagnosis of small hepatic nodules such as regenerative nodules, focal nodular hyperplasia (FNH)/FNH-like lesions, dysplastic nodules, early HCCs, advanced HCCs, and so on, on the basis of the tumor vessel pattern (centrifugal and centripetal) and enhancement pattern on CEUS using Sonazoid and HBP EOBMRI findings. Dr. Hironori Tanaka explained the use of B-mode, color/power Doppler including SMI, and CEUS using Sonazoid including techniques such as defect reperfusion imaging, maximum intensity projection, and so on for the diagnosis, histological grading, and evaluation of treatment efficacy of mainly typical HCCs. He also explained how to use 3D-GPS markers for the ablation treatment of HCCs using transabdominal US. Dr. Yasunori Minami explained in detail how to use $\mathrm{CT}$ or MRI/US and US/US fusion imaging for RFA treatment of HCCs. He also clearly explained the advantages and 
disadvantages of US fusion imaging technologies. Dr. Kento Imajo clearly explained the advantages and disadvantages of MWA. Additionally, he provided a detailed comparison of MWA with RFA for treatment of HCCs. I believe these four review articles will contribute to better diagnosis and treatment of HCCs.

\section{References}

1. Bray F, Ferlay J, Soerjomataram I, et al. Global cancer statistics 2018: GLOBOCAN estimates of incidence and mortality worldwide for 36 cancers in 185 countries. CA Cancer J Clin. 2018;68:394-424.

2. Kudo M. Management of hepatocellular carcinoma in Japan as a world-leading model. Liver Cancer. 2018;7:134-47.

3. Kudo M, Lencioni R, Marrero JA, et al. Regional differences in sorafenib-treated patients with hepatocellular carcinoma: GIDEON observational study. Liver Int. 2016;36:1196-205.

4. Kudo M, Ikeda M, Takayama T, et al. Safety and efficacy of sorafenib in Japanese patients with hepatocellular carcinoma in clinical practice: a subgroup analysis of GIDEON. J Gastroenterol. 2016;51:1150-60.

5. Numata K, Morimoto M, Ogura T, et al. Ablation therapy guided by contrast-enhanced sonography with Sonazoid for hepatocellular carcinoma lesions not detected by conventional sonography. J Ultrasound Med. 2008;27:395-406.

6. Kunishi Y, Numata K, Morimoto M, et al. Efficacy of fusion imaging combining sonography and hepatobiliary phase MRI with GdEOB-DTPA to detect small hepatocellular carcinoma. AJR Am J Roentgenol. 2012;198:106-14.

7. Yang F, Zhao J, Liu C, et al. Superb microvascular imaging technique in depicting vascularity in focal liver lesions: more hypervascular supply patterns were depicted in hepatocellular carcinoma. Cancer Imaging. 2019;19:92.
8. Numata K, Fukuda H, Morimoto M, et al. Use of fusion imaging combining contrast-enhanced ultrasonography with a perflubutane-based contrast agent and contrast-enhanced computed tomography for the evaluation of percutaneous radiofrequency ablation of hypervascular hepatocellular carcinoma. Eur J Radiol. 2012;81:2746-53.

9. Nishigori S, Numata K, Irie K, et al. Fusion imaging with contrast-enhanced ultrasonography for evaluating the early therapeutic efficacy of radiofrequency ablation for small hypervascular hepatocellular carcinomas with iso-echoic or unclear margins on conventional ultrasonography. J Med Ultrason. 2018;45:405-15.

10. Minami Y, Minami T, Hagiwara S, et al. Ultrasound-ultrasound image overlay fusion improves real-time control of radiofrequency ablation margin in the treatment of hepatocellular carcinoma. Eur Radiol. 2018;28:1986-93.

11. Xu EJ, Lv SM, Li K, et al. Immediate evaluation and guidance of liver cancer thermal ablation by three-dimensional ultrasound/ contrast-enhanced ultrasound fusion imaging. Int J Hyperthermia. 2018;34:870-6.

12. Sanga K, Numata $K$, Nihonmatsu $H$, et al. Use of intra-procedural fusion imaging combining contrast-enhanced ultrasound using a perflubutane-based contrast agent and auto sweep three-dimensional ultrasound for guiding radiofrequency ablation and evaluating its efficacy in patients with hepatocellular carcinoma. Int J Hyperthermia. 2020;37:202-11.

13. Seo J, Koizumi N, Funamoto T, et al. Visual serving for a US guided therapeutic HIFU system by coagulated lesion tracking: a phantom study. Int J Med Robot Comput Assist Surg. 2011;7:237-47.

14. Imajo K, Tomeno W, Kanezaki M, et al. New microwave ablation system for unresectable liver tumors that forms large, spherical ablation zones. J Gastroenterol Hepatol. 2018;33:2007-144.

Publisher's Note Springer Nature remains neutral with regard to jurisdictional claims in published maps and institutional affiliations. 